\title{
Online Risk-Averse Submodular Maximization
}

\author{
Tasuku Soma $^{1,2}$, Yuichi Yoshida ${ }^{3}$ \\ ${ }^{1}$ The University of Tokyo \\ ${ }^{2}$ Massachusetts Institute of Technology \\ ${ }^{3}$ National Institute of Informatics \\ tasuku_soma@mist.i.u-tokyo.ac.jp, tasuku@mit.edu, yyoshida@nii.ac.jp
}

\begin{abstract}
We present a polynomial-time online algorithm for maximizing the conditional value at risk $(\mathrm{CVaR})$ of a monotone stochastic submodular function. Given $T$ i.i.d. samples from an underlying distribution arriving online, our algorithm produces a sequence of solutions that converges to a $(1-1 / e)$-approximate solution with a convergence rate of $O\left(T^{-1 / 4}\right)$ for monotone continuous DR-submodular functions. Compared with previous offline algorithms, which require $\Omega(T)$ space, our online algorithm only requires $O(\sqrt{T})$ space. We extend our online algorithm to portfolio optimization for monotone submodular set functions under a matroid constraint. Experiments conducted on real-world datasets demonstrate that our algorithm can rapidly achieve CVaRs that are comparable to those obtained by existing offline algorithms.
\end{abstract}

\section{Introduction}

Submodular function maximization is a ubiquitous problem naturally arising in broad areas such as machine learning, social network analysis, economics, combinatorial optimization, and decision-making [Krause and Golovin, 2014; Buchbinder and Feldman, 2018]. Submodular maximization in these applications is stochastic in nature, i.e., the input data could be a sequence of samples drawn from some underlying distribution, or there could be some uncertainty in the environment. In this paper, we consider nonnegative monotone continuous DR-submodular ${ }^{1}$ [Bian et al., 2017] functions $F(\boldsymbol{x} ; z)$ parameterized by a random variable $z$ drawn from some distribution $\mathcal{D}$. The simplest approach for such stochastic submodular objectives is to maximize the expectation $\mathbf{E}_{z \sim \mathcal{D}}[F(\boldsymbol{x} ; z)]$, which has been extensively studied [Karimi et al., 2017; Hassani et al., 2017; Mokhtari et al., 2018; Karbasi et al., 2019].

However, in real-world decision-making tasks in finance, robotics, and medicine, we sometimes must be risk-averse: We want to minimize the risk of suffering a considerably small gain rather than simply maximizing the expected

\footnotetext{
${ }^{1}$ See Section 2 for the definition of continuous DRsubmodularity.
}

gain [Mansini et al., 2007; Yau et al., 2011; Tamar et al., 2015]. In medical applications, for example, we must avoid catastrophic events such as patient fatalities. In finance and robotics, all progress ceases when poor decisions cause bankruptcies or irreversible damage to robots.

Conditional value at risk (CVaR) is a popular objective for such risk-averse domains [Rockafellar and others, 2000; Krokhmal et al., 2002]. Formally, given a parameter $\alpha \in$ $[0,1]$, the $\mathrm{CVaR}$ of a feasible solution $\boldsymbol{x} \in \mathbb{R}^{n}$ is defined as

$$
\mathrm{CVaR}_{\alpha, \mathcal{D}}(\boldsymbol{x})=\underset{z \sim \mathcal{D}}{\mathbf{E}}\left[F(\boldsymbol{x} ; z) \mid F(\boldsymbol{x} ; z) \leq \operatorname{VaR}_{\alpha, \mathcal{D}}(\boldsymbol{x})\right],
$$

where $\operatorname{VaR}_{\alpha, \mathcal{D}}(X)$ is the $\alpha$-quantile of the random variable $F(\boldsymbol{x} ; z)$, i.e.,

$$
\operatorname{VaR}_{\alpha, \mathcal{D}}(X)=\sup \left\{\tau \in \mathbb{R}: \operatorname{Pr}_{z \sim \mathcal{D}}(F(\boldsymbol{x} ; z) \leq \tau) \leq \alpha\right\} .
$$

Note that when $\alpha=1$, the CVaR is simply the expected value of $F(\boldsymbol{x} ; z)$. Since $\alpha$ is typically set to be 0.10 or 0.05 in practice, we assume that $\alpha$ is a fixed constant throughout this paper. When $\alpha$ is clear from the context, we omit it from the notations. CVaR can also be characterized by a variational formula:

$$
\mathrm{CVaR}_{\mathcal{D}}(\boldsymbol{x})=\max _{\tau \in[0,1]} \tau-\frac{1}{\alpha} \underset{z \sim \mathcal{D}}{\mathbf{E}}[\tau-F(\boldsymbol{x} ; z)]_{+},
$$

where $[\cdot]_{+}=\max \{\cdot, 0\}$ [Rockafellar and others, 2000].

[Maehara, 2015] initiated the study of maximizing the CVaR of stochastic submodular set functions. It was shown that the CVaR of a stochastic submodular set function is not necessarily submodular, and that it is impossible to compute a single set that attains any multiplicative approximation to the optimal CVaR. [Ohsaka and Yoshida, 2017] introduced a relaxed problem of finding a portfolio over sets rather than a single set, and devised the first CVaR maximization algorithm with an approximation guarantee for the influence maximization problem [Kempe et al., 2003], a prominent example of discrete submodular maximization. [Wilder, 2018] further considered this approach and devised an algorithm called RASCAL for maximizing the CVaR of continuous DR-submodular functions subject to a down-closed convex set.

The algorithms mentioned above are offline (batch) meth$o d s$, i.e., a set of samples drawn from the underlying distribution $\mathcal{D}$ is given as the input. However, because the number of 
samples needed to accurately represent $\mathcal{D}$ can be exceedingly large, it is often inefficient or even impossible to store all the samples in memory. Further, when a new sample is observed, these offline algorithms must be rerun from scratch.

In the machine learning community, online methods, which can efficiently handle large volumes of data, have been extensively studied [Hazan, 2016]. Online methods read data in a streaming fashion, and update the current solution using a few data elements stored in memory. Further, online methods can update a solution at a low computational cost.

\subsection{Our Contributions}

In this work, we propose online algorithms for maximizing the CVaR of stochastic submodular objectives in continuous and discrete settings.

Continuous setting. Let $z_{1}, \ldots, z_{T}$ be i.i.d. samples drawn from $\mathcal{D}$ arriving sequentially and $K \subseteq \mathbb{R}^{n}$ be a down-closed convex set. Our main result is a polynomial-time online algorithm, STOCHASTICRASCAL, that finds $\boldsymbol{x} \in K$ such that

$$
\mathbf{E}\left[\mathrm{CVaR}_{\mathcal{D}}(\boldsymbol{x})\right] \geq\left(1-\frac{1}{e}\right) \operatorname{CVaR}_{\mathcal{D}}\left(\boldsymbol{x}^{*}\right)-O\left(T^{-1 / 4}\right),
$$

for any $x^{*} \in K$, where the expectation is taken over $z_{1}, \ldots, z_{T}$ and the randomness of the algorithm. STOCHASTICRASCAL only stores $O(\sqrt{T})$ data in memory, drawing a contrast to RASCAL, which store all $T$ data points. Note that the approximation ratio of $1-1 / e$ is optimal for any algorithm that performs polynomially many function value queries even if $\alpha=1$ [Vondrák, 2013]. We also conduct several experiments on real-world datasets to show the practical efficiency of our algorithm. We demonstrate that our algorithm rapidly achieves CVaR comparable to that obtained by known offline methods.

Discrete setting. As an application of the above algorithm, we devise an online algorithm to create a portfolio that maximizes the CVaR of a discrete submodular function subject to a matroid constraint. Let $f(X ; z): 2^{V} \rightarrow[0,1]$ be a monotone stochastic submodular set function on a ground set $V$ and $\mathcal{I} \subseteq 2^{V}$ be a matroid. The goal is to find a portfolio $\mathcal{P}$ of feasible sets in $\mathcal{I}$ that maximizes

$$
\mathrm{CVaR}_{\mathcal{D}}(\mathcal{P})=\max _{\tau \in[0,1]} \tau-\frac{1}{\alpha} \underset{z \sim \mathcal{D}}{\mathbf{E}}[\tau-\underset{X \sim \mathcal{P}}{\mathbf{E}} f(X ; z)]_{+},
$$

given i.i.d. samples from $\mathcal{D}$. We show that this problem can be reduced to online CVaR maximization of continuous DRsubmodular functions on a matroid polytope, and devise a polynomial-time online approximation algorithm for creating a portfolio $\mathcal{P}$ such that

$$
\mathbf{E}\left[\operatorname{CVaR}_{\mathcal{D}}(\mathcal{P})\right] \geq\left(1-\frac{1}{e}\right) \operatorname{CVaR}_{\mathcal{D}}\left(\mathcal{P}^{*}\right)-O\left(T^{-1 / 10}\right)
$$

for any portfolio $\mathcal{P}^{*}$ over feasible sets. Note that this algorithm is the first online algorithm that converges to a $(1-$ $1 / e)$-approximation portfolio in the discrete setting, which generalizes the known offline algorithm [Wilder, 2018] to the i.i.d. setting.

\subsection{Our Techniques}

To analyze our online algorithms, we introduce a novel adversarial online learning problem, which we call adversarial online submodular CVaR learning. This online learning problem is described as follows. For $t=1, \ldots, T$, the learner chooses $\boldsymbol{x}_{t} \in K$ and $\tau_{t} \in[0,1]$ possibly in a randomized manner. After $\boldsymbol{x}_{t}$ and $\tau_{t}$ are chosen, the adversary reveals a monotone continuous DR-submodular function $F_{t}: K \rightarrow[0,1]$ to the learner. The goal of the learner is to minimize the approximate regret

$\operatorname{regret}_{1-1 / e}(T)=\left(1-\frac{1}{e}\right) \sum_{t=1}^{T} H_{t}\left(\boldsymbol{x}^{*}, \tau^{*}\right)-\sum_{t=1}^{T} H_{t}\left(\boldsymbol{x}_{t}, \tau_{t}\right)$

for arbitrary $\boldsymbol{x}^{*} \in K$ and $\tau^{*} \in[0,1]$, where the function $H_{t}$ is given by

$$
H_{t}(\boldsymbol{x}, \tau)=\tau-\frac{1}{\alpha}\left[\tau-F_{t}(\boldsymbol{x})\right]_{+} .
$$

We devise an efficient algorithm that achieves $O\left(T^{3 / 4}\right)$ approximate regret in expectation. Further, we show that, given an online algorithm with a sublinear approximate regret, we can construct an online algorithm that achieves a $(1-1 / e)$ approximation to $\mathrm{CVaR}$ maximization, whose convergence rate is $\mathbf{E}\left[\right.$ regret $\left._{1-1 / e}(T)\right] / T$. Combining these results, we obtain an online $(1-1 / e)$-approximation algorithm for $\mathrm{CVaR}$ maximization with a convergence rate of $O\left(T^{-1 / 4}\right)$.

We remark that adversarial online submodular CVaR learning may be of interest in its own right: Although the objective function $H_{t}$ is neither monotone nor continuous DRsubmodular in general, we can design an online algorithm with a sublinear $(1-1 / e)$-regret by exploiting the underlying structure of $H_{t}$. As per our knowledge, an online algorithm for non-monotone and non-DR-submodular maximization does not exist in the literature.

\subsection{Related Work}

Several studies focused on CVaR optimization in the adversarial online settings and i.i.d. settings. [Tamar et al., 2015] studied CVaR optimization over i.i.d. samples and analyzed stochastic gradient descent under the strong assumption that CVaR is continuously differentiable. Recently, [Cardoso and $\mathrm{Xu}, 2019]$ introduced the concept of the CVaR regret for convex loss functions and provided online algorithms for minimizing the CVaR regret under bandit feedback.

Online and stochastic optimization of submodular maximization have been extensively studied in [Streeter and Golovin, 2008; Streeter et al., 2009; Golovin et al., 2014; Karimi et al., 2017; Hassani et al., 2017; Mokhtari et al., 2018; Chen et al., 2018; Roughgarden and Wang, 2018; Soma, 2019; Karbasi et al., 2019; Zhang et al., 2019]. These studies optimize either the approximate regret or the expectation and do not consider CVaR.

Another line of related work is robust submodular maximization [Krause et al., 2008; Chen et al., 2017; Anari et $a l ., 2019]$. In robust submodular maximization, we maximize the minimum of $N$ submodular functions, i.e., $\min _{i=1}^{N} f_{i}(X)$. Robust submodular maximization is the limit of CVaR maximization, where $\mathcal{D}$ is the uniform distribution over $N$ values 
and $\alpha \rightarrow 0$. Recently, [Staib et al., 2019] proposed distributionally robust submodular optimization, which maximizes $\min _{\mathcal{D} \in P} \mathbf{E}_{z \sim \mathcal{D}} f(X ; z)$ for an uncertainty set $P$ of distributions. It is known that CVaR can be formulated in the distributionally robust framework [Shapiro et al., 2014]. However, the algorithms proposed by [Staib et al., 2019] require that $P$ is a subset of the $N$-dimensional probability simplex; moreover, their time complexity depends on $N$. Our algorithms work even if $N$ is infinite.

\subsection{Organization of This Paper}

This paper is organized as follows. Section 2 introduces the background of submodular optimization. Sections 3 and 4 describe our algorithms for continuous and discrete setting, respectively. Section 5 present experimental results using real-world dataset. The omitted analysis and the details of adversarial setting can be found in the full version.

\section{Preliminaries}

Throughout the paper, $V$ denotes the ground set and $n$ denotes the size of the ground set. For a set function $f: 2^{V} \rightarrow$ $\mathbb{R}$, the multilinear extension $F:[0,1]^{V} \rightarrow \mathbb{R}$ is defined as $F(\boldsymbol{x})=\sum_{S \subseteq V} f(S) \prod_{i \in S} x_{i} \prod_{i \notin S}\left(1-x_{i}\right)$. For a matroid on $V$, the base polytope is the convex hull of bases of the matroid. It is well-known that the linear optimization on a base polytope can be solved by the greedy algorithm [Fujishige, 2005].

We denote the Euclidean norm and inner product by $\|\cdot\|$ and $\langle\cdot, \cdot\rangle$, respectively. The $\ell^{p}$ norm $(1 \leq p \leq \infty)$ is denoted by $\|\cdot\|_{p}$. The Euclidean projection of $\boldsymbol{x}$ onto a set $K$ is denoted by $\operatorname{proj}_{K}(\boldsymbol{x})$. A convex set $K \subseteq \mathbb{R}_{\geq 0}^{n}$ is said to be down-closed if $\boldsymbol{y} \in K$ and $\mathbf{0} \leq \boldsymbol{x} \leq \boldsymbol{y}$ imply $\boldsymbol{x} \in K$. A function $f: \mathbb{R}^{n} \rightarrow \mathbb{R}$ is said to be L-Lipschitz (continuous) for $L>0$ if $|f(\boldsymbol{x})-f(\boldsymbol{y})| \leq L\|\boldsymbol{x}-\boldsymbol{y}\|$ for all $\boldsymbol{x}, \boldsymbol{y}$. We say that $f$ is $\beta$-smooth for $\beta>0$ if $f$ is continuously differentiable and $\|\nabla f(\boldsymbol{x})-\nabla f(\boldsymbol{y})\| \leq \beta\|\boldsymbol{x}-\boldsymbol{y}\|$. A smooth function $F: \mathbb{R}^{n} \rightarrow \mathbb{R}$ is said to be continuous DR-submodular [Bian et al., 2017] if $\frac{\partial^{2} F}{\partial x_{i} \partial x_{j}} \leq 0$ for all $i, j$. The multilinear extension of a submodular function is known to be DR-submodular [Calinescu et al., 2011]. The continuous DR-submodularity implies up-concavity: For a continuous DR-submodular function $F, \boldsymbol{x} \in \mathbb{R}^{n}$, and $\boldsymbol{d} \geq \mathbf{0}$, the univariate function $t \mapsto F(\boldsymbol{x}+t \boldsymbol{d})$ is concave.

The uniform distribution of a set $K$ is denoted by $\operatorname{Unif}(K)$. The standard normal distribution is denoted by $N(\mathbf{0}, I)$.

\section{CVaR Maximization of Continuous DR-submodular Functions}

We present our online algorithm for $\mathrm{CVaR}$ maximization via i.i.d. samples. Let $F_{t}: \mathbb{R}^{n} \rightarrow[0,1]$ be the monotone continuous DR-submodular function corresponding to the $t$-th sample $z_{t}$, i.e.,

$$
F_{t}(\boldsymbol{x})=F\left(\boldsymbol{x} ; z_{t}\right)
$$

for $t=1, \ldots, T$. Similarly, define an auxiliary function $H_{t}$ with respect to $z_{t}$ by

$$
H_{t}(\boldsymbol{x}, \tau)=\tau-\frac{1}{\alpha}\left[\tau-F_{t}(\boldsymbol{x})\right]_{+}=\tau-\frac{1}{\alpha}\left[\tau-F\left(\boldsymbol{x} ; z_{t}\right)\right]_{+} .
$$

for $t=1, \ldots, T$. Let $K \subseteq \mathbb{R}_{>0}^{n}$ be a down-closed convex set. Formally, we make the following very mild assumptions on $F_{t}$ and $K$.

\section{Assumption 1.}

- For all $t, F_{t}$ is $L$-Lipschitz and $\beta$-smooth, and $\left\|\nabla F_{t}\right\| \leq$ $G$.

- The diameter of $K$ is bounded by $D$.

- We are given a linear optimization oracle over $K$.

When the underlying norm is the $\ell^{p}$-norm with $p \neq 2$, we write $G_{p}$ to emphasize it. For example, if $F_{t}$ is the multilinear extension of a submodular set function $f_{t}: 2^{V} \rightarrow[0,1]$ and $K$ is the base polytope of a rank- $k$ matroid, we have $L=\beta=$ $O(n), G_{\infty}=1, D=O(\sqrt{k})$.

Our algorithm borrows some ideas from an algorithm called RASCAL [Wilder, 2018]. First, we define a smoothed auxiliary function $\tilde{H}_{t}:[0,1]^{V} \times[0,1] \rightarrow[0,1]$ as

$$
\tilde{H}_{t}(\boldsymbol{x}, \tau)=\frac{1}{u} \int_{0}^{u}\left(\tau+\xi-\frac{1}{\alpha}\left[\tau+\xi-F_{t}(\boldsymbol{x})\right]_{+}\right) d \xi
$$

where $u>0$ is a smoothing parameter specified later. This smoothing guarantees that $\tilde{H}_{t}$ is differentiable for all $\boldsymbol{x}$ and has Lipschitz continuous gradients.

Lemma 2 (Lemma 6 of [Wilder, 2018]).

1. $\left|H_{t}(\boldsymbol{x}, \tau)-\tilde{H}_{t}(\boldsymbol{x}, \tau)\right| \leq \frac{u(1+1 / \alpha)}{2}$ for all $\boldsymbol{x}$ and $\tau$.

2. If $F_{t}$ is L-Lipschitz and $\beta$-smooth, and $\left\|\nabla F_{t}\right\| \leq G$, then $\nabla_{\boldsymbol{x}} \tilde{H}_{t}$ is $\frac{1}{\alpha}\left(\beta+\frac{L G}{u}\right)$-Lipschitz.

Lemma 3 ([Wilder, 2018]). The function $\max _{\tau} \tilde{H}_{t}(\cdot, \tau)$ is monotone and up-concave.

\subsection{StochasticRASCAL}

We now formally describe our algorithm, STOCHASTICRASCAL. We note that RASCAL runs the Frank-Wolfe algorithm on a function $\max _{\tau} \sum_{t=1}^{T} \tilde{H}_{t}(\cdot, \tau)$. Owing to the up-concavity and smoothness properties of this function, one can obtain $(1-1 / e)$-approximation. However, in our online setting, we cannot evaluate this function because $\tilde{H}_{t}$ will be revealed online, and hence we cannot simply run RASCAL.

To overcome the issue above, first we split the $T$ samples into mini-batches of length $B$, which we specify later. The key idea is to use the following objective function

$$
\bar{H}_{b}(\boldsymbol{x})=\max _{\tau} \frac{1}{B} \sum_{t=(b-1) B+1}^{b B} \tilde{H}_{t}(\boldsymbol{x}, \tau)
$$

for each $b$-th mini-batch $(b=1, \ldots, T / B)$. We can see that $\bar{H}_{b}(\boldsymbol{x})$ is monotone, up-concave, and can be evaluated only using samples in the $b$-th mini-batch. Then, we run a perturbed version of Frank-Wolfe algorithm [Golovin et al., 2014; Bian et al., 2017] on $\bar{H}_{b}$. More formally, we first initialize $\boldsymbol{x}_{b+1}^{0}=\mathbf{0}$ and for each $s=0, \delta, 2 \delta, \ldots, 1-\delta$, we perform the update

$$
\boldsymbol{x}_{b}^{s+\delta} \leftarrow \boldsymbol{x}_{b}^{s}+\delta \boldsymbol{d}_{b}^{s}
$$



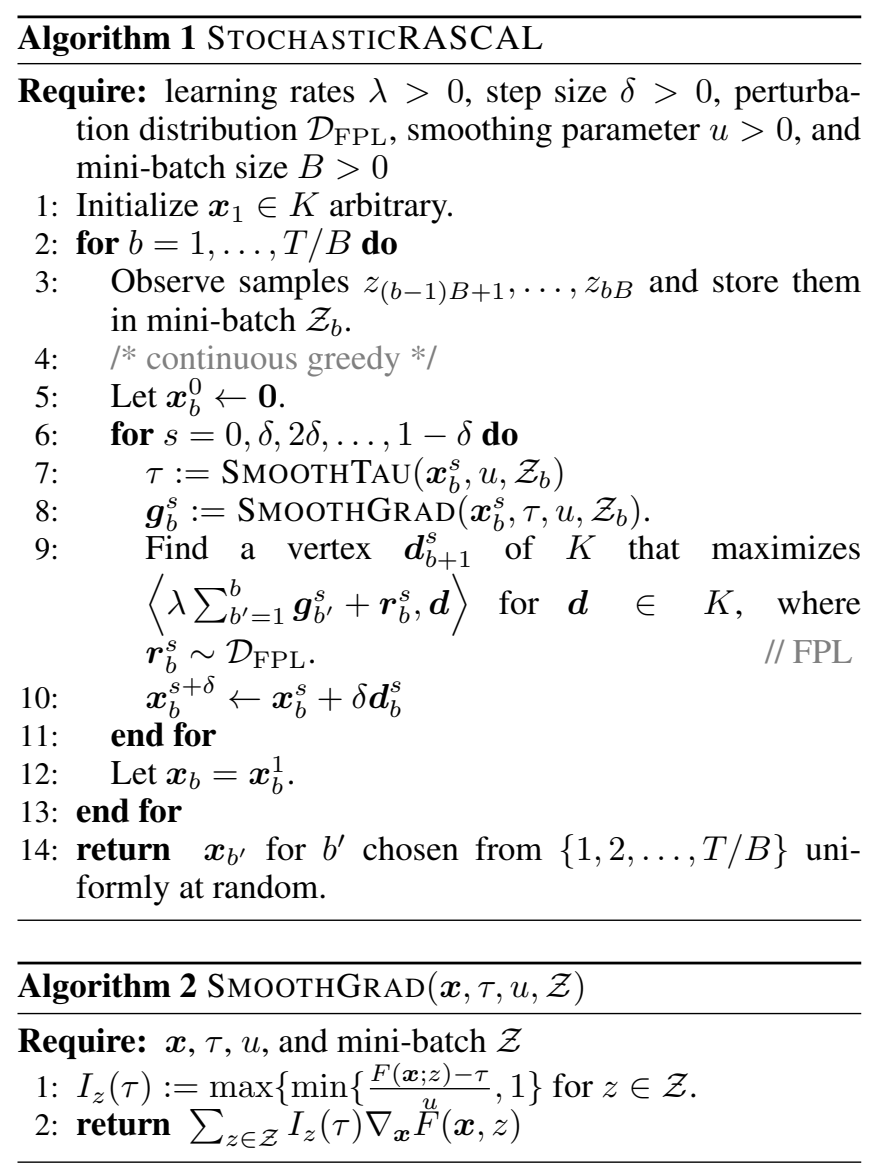

where $\delta$ is the step size, $\boldsymbol{d}_{b}^{s}$ is a solution to a perturbed linear optimization problem on $K$ :

$$
\boldsymbol{d}_{b}^{s} \in \underset{\boldsymbol{d} \in K}{\operatorname{argmax}}\left\langle\lambda \sum_{b^{\prime}=1}^{b} \nabla \bar{H}_{b^{\prime}}\left(\boldsymbol{x}_{b^{\prime}}^{s}\right)+\boldsymbol{r}_{b}^{s}, \boldsymbol{d}\right\rangle .
$$

Here, $\boldsymbol{r}_{b}^{s} \sim \mathcal{D}_{\mathrm{FPL}}$ is a perturbation vector. This perturbation trick aims to stabilize the algorithm so that we can maximize the true objective $\max _{\tau} \sum_{t=1}^{T} \tilde{H}_{t}(\cdot, \tau)$ using only mini-batch objectives.

In each iteration of continuous greedy, we need the gradients $\nabla \bar{H}_{b}\left(\boldsymbol{x}_{b}^{s}\right)$, which in turn requires us to compute $\operatorname{argmax}_{\tau} \frac{1}{B} \sum_{t=(b-1) B+1}^{b B} \tilde{H}_{t}\left(\boldsymbol{x}_{b}^{s}, \tau\right)$. These gradients and the optimal $\tau$ can be computed by SMOOTHGRAD and SmoothTAU subroutines, respectively, which were proposed in [Wilder, 2018]; See Algorithms 2 and 3.

Let us write $\boldsymbol{x}_{b}:=\boldsymbol{x}_{b}^{1}$ for $b=1, \ldots, T / B$. The final output of STOCHASTICRASCAL is $\boldsymbol{x}_{b^{\prime}}$ for a random index $b^{\prime}$ chosen uniformly at random from $\{1,2, \ldots, T / B\}$. The pseudocode of STOCHASTICRASCAL is presented in Algorithm 1.

\subsection{Convergence Rate via Regret Bounds}

Let us consider the convergence rate of STOCHASTICRASCAL. The main challenge of the analysis is how to set the parameters used in the algorithm, i.e., learning rates $\lambda$, step size $\delta$, perturbation distribution $\mathcal{D}_{\mathrm{FPL}}$, smoothing parameter

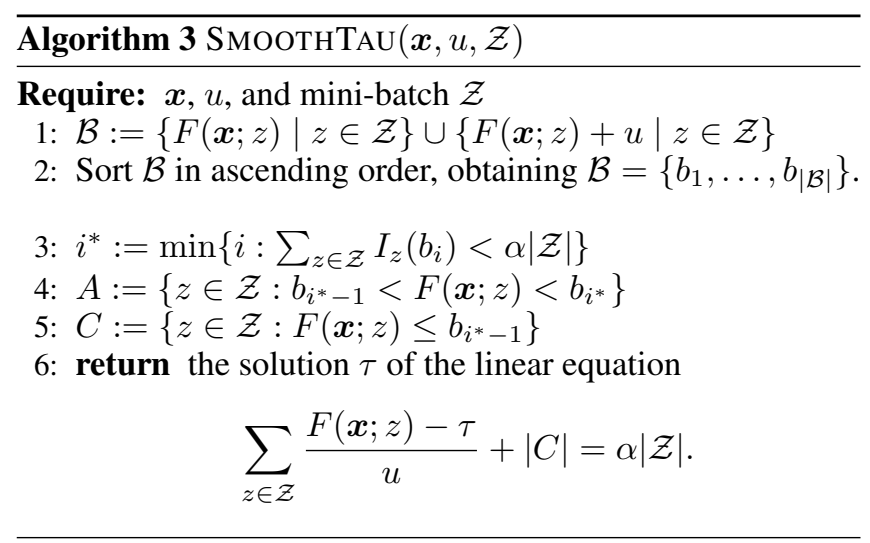

$u$, and mini-batch size $B$, to achieve the desired $O\left(T^{-1 / 4}\right)$ convergence rate.

To this end, using tools from online convex optimization, we prove an approximate regret bound for a variant of STOCHASTICRASCAL for adversarial online submodular CVaR learning (see Introduction for the definition).

Theorem 4 (informal). There exists an efficient online algorithm for adversarial online CVaR learning with

$$
\left(1-\frac{1}{e}\right) \sum_{t=1}^{T} H_{t}\left(\boldsymbol{x}^{*}, \tau^{*}\right)-\mathbf{E}\left[\sum_{t=1}^{T} H_{t}\left(\boldsymbol{x}_{t}, \tau_{t}\right)\right]=O\left(T^{3 / 4}\right)
$$

for an arbitrary $\boldsymbol{x}^{*} \in K$ and $\tau^{*} \in[0,1]$, where the big-O notation hides a factor polynomial in $\alpha, \beta, D, G$, and $n$.

We then show that the above regret bound can be used to show a convergence rate of STOCHASTICRASCAL. The technical detail of the adversarial setting and the proof of the following theorem is deferred to the full version.

Theorem 5. Under Assumption 1, STOCHASTICRASCAL outputs $\boldsymbol{x} \in K$ such that for any $\boldsymbol{x}^{*} \in K$,

$$
\begin{aligned}
& \mathbf{E}\left[\operatorname{CVaR}_{\mathcal{D}}(\boldsymbol{x})\right] \\
& \geq\left(1-\frac{1}{e}\right) \operatorname{CVaR}_{\mathcal{D}}\left(\boldsymbol{x}^{*}\right)-O\left(\frac{\sqrt{C_{\alpha} G D} n^{1 / 8}}{\sqrt{\alpha}}\right) T^{-1 / 4},
\end{aligned}
$$

where we set $B=\frac{\alpha C_{\alpha} \sqrt{T}}{D G n^{1 / 4}}, \delta=\frac{\alpha^{2}}{D^{2}\left((1+\alpha) G L \sqrt{T}+\alpha \beta T^{1 / 4}\right)}$, $\lambda=\frac{\alpha D n^{1 / 4} \sqrt{B / T}}{G}, u=\frac{T^{-1 / 4}}{(1+1 / \alpha)}$, and $\mathcal{D}_{\mathrm{FPL}}=\operatorname{Unif}\left([0,1]^{n}\right)$, and $C_{\alpha}:=\max \left\{1, \frac{1}{\alpha}-1\right\}$. Further, if $K$ is an integral polytope contained in $\left\{\boldsymbol{x} \in[0,1]^{n}: \sum_{i} x_{i}=k\right\}$, then

$$
\begin{aligned}
\mathbf{E}\left[\operatorname{CVaR}_{\mathcal{D}}(\boldsymbol{x})\right] \geq & \left(1-\frac{1}{e}\right) \operatorname{CVaR}_{\mathcal{D}}\left(\boldsymbol{x}^{*}\right) \\
& -O\left(\frac{\sqrt{C_{\alpha} G_{\infty}} k^{3 / 4} \log ^{1 / 4} n}{\sqrt{\alpha}}\right) T^{-1 / 4}
\end{aligned}
$$

for $B=\frac{\sqrt{2} C_{\alpha} \sqrt{T} \alpha}{2 G_{\infty} k^{3 / 2} \sqrt{\log (n)}}, \quad \delta=\frac{\alpha^{2}}{D^{2}\left((1+\alpha) G L \sqrt{T}+\alpha \beta T^{1 / 4}\right)}$, $\lambda=\sqrt{\frac{B}{T k}}, u=\frac{T^{-1 / 4}}{(1+1 / \alpha)}$, and $\mathcal{D}_{\mathrm{FPL}}=N(\mathbf{0}, I)$.

To achieve $\mathbf{E}\left[\operatorname{CVaR}_{\mathcal{D}}(\boldsymbol{x})\right] \geq\left(1-\frac{1}{e}\right) \operatorname{CVaR}_{\mathcal{D}}\left(\boldsymbol{x}^{*}\right)-\varepsilon$ for a desired error $\varepsilon>0$, STOCHASTICRASCAL requires 
$O\left(\frac{D^{2} G^{2} \sqrt{n}}{\varepsilon^{4}}\right)$ samples and $O\left(\frac{D G n^{1.25}}{\varepsilon^{2}}\right)$ space, whereas RASCAL [Wilder, 2018] requires $O\left(\frac{n}{\varepsilon^{2}}\right)$ samples and $O\left(\frac{n^{2}}{\varepsilon^{2}}\right)$ space. Our algorithm runs in a smaller space when the parameters are of moderate size. For example, if $\beta=D=O(1)$ and $L=G=o\left(n^{1 / 4}\right)$, the space complexity of STOCHASTICRASCAL is better than that of RASCAL.

\section{CVaR Maximization of Discrete Submodular Functions}

We now present our online algorithm for a monotone submodular set function and a matroid constraint. Let $f_{t}$ : $2^{V} \rightarrow[0,1]$ be a monotone submodular function corresponding to the $t$-th sample and $F_{t}$ be its multilinear extension for $t=1, \ldots, T$.

The basic idea is to run STOCHASTICRASCAL on the multilinear extensions $F_{t}$ and the matroid polytope $K$. However, we must address several technical obstacles. First, we must compare the output portfolio with the optimal portfolio; the error bound in the previous sections compared it with the optimal solution. To this end, we make multiple copies of variables so that we can approximate an optimal portfolio by a uniform distribution over a multiset of feasible solutions. More precisely, we define a continuous DR-submodular function $\vec{F}_{t}: K^{r} \rightarrow[0,1]$ by

$$
\bar{F}_{t}\left(\boldsymbol{x}^{1}, \ldots, \boldsymbol{x}^{r}\right)=\frac{1}{r} \sum_{i=1}^{r} F_{t}\left(\boldsymbol{x}^{i}\right)
$$

for some sufficiently large $r$. Then, we feed $\bar{F}_{1}, \ldots, \bar{F}_{T}$ to StochasticRASCAL. Suppose that we obtain $\left(\boldsymbol{x}_{b}^{1}, \ldots, \boldsymbol{x}_{b}^{r}\right)$ at Line 12 for each mini-batch $b=1, \ldots, T / B$. Abusing the notation, let us denote $\left(\boldsymbol{x}_{t}^{1}, \ldots, \boldsymbol{x}_{t}^{r}\right):=\left(\boldsymbol{x}_{b}^{1}, \ldots, \boldsymbol{x}_{b}^{r}\right)$ when the $t$-th sample is in the $b$-th mini-batch.

Next, we need to convert $\boldsymbol{x}_{t}^{1}, \ldots, \boldsymbol{x}_{t}^{r}$ to feasible sets without significantly deteriorating the values of the multilinear extensions. To this end, we independently apply randomized swap rounding [Chekuri et al., 2010] $q$ times to each $\boldsymbol{x}^{i}$ to obtain feasible sets $X_{t}^{i, 1}, \ldots, X_{t}^{i, q}$. Note that randomized swap rounding is oblivious rounding and independent from $F_{t}$. We can show that $\frac{1}{q} \sum_{j=1}^{q} f_{t}\left(X_{t}^{i, j}\right)$ is close to $F_{t}\left(\boldsymbol{x}_{t}^{i}\right)$ by using a concentration inequality. Finally, after $T$ rounds, we return the uniform portfolio over all $X_{t}^{i, q}$. The pseudocode is given in Algorithm 4. Carefully choosing $r$ and $q$, we obtain the following theorem.

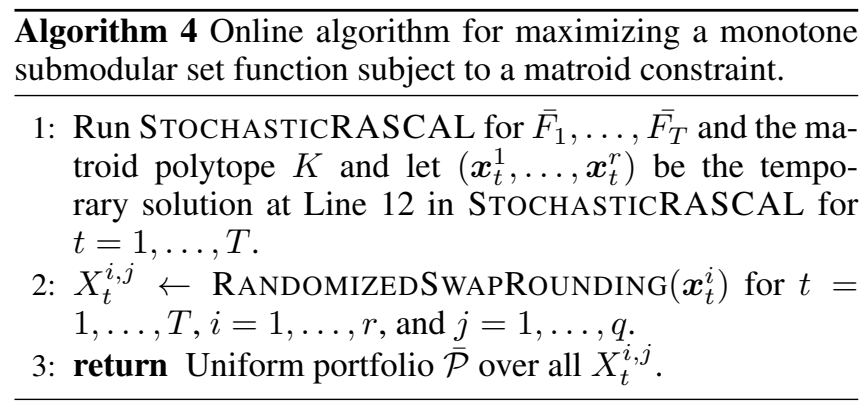

Theorem 6. Algorithm 4 achieves

$$
\begin{aligned}
& \mathbf{E}[\operatorname{CVaR}(\overline{\mathcal{P}})] \\
& \geq\left(1-\frac{1}{e}\right) \operatorname{CVaR}_{\mathcal{D}}\left(\mathcal{P}^{*}\right)-O\left(k^{3 / 4} \log ^{1 / 4}(n) T^{-1 / 10}\right)
\end{aligned}
$$

for arbitrary portfolio $\mathcal{P}^{*}$, where we set $r=\tilde{O}\left(T^{1 / 5}\right)$ and $q=\tilde{O}\left(T^{3 / 4}\right)$ and the expectation is taken over $z_{1}, \ldots, z_{T}$ and the randomness of the algorithm.

\section{Experiments}

In this section, we show our experimental results. In all the experiments, the parameter $\alpha$ of CVaR was set to 0.1 . The experiments were conducted on a Linux server with Intel Xeon Gold $6242(2.8 \mathrm{GHz})$ and 384GB of main memory.

Problem Description. We conducted experiments on the sensor resource allocation problem, in which the goal is to rapidly detect a contagion spreading through a network using a limited budget [Bian et al., 2017; Leskovec et al., 2007; Soma and Yoshida, 2015].

Here, we follow the configuration of the experiments conducted in [Wilder, 2018]. Let $G=(V, E)$ be a graph on $n$ vertices. A contagion starts at a random vertex and spreads over time according to some specific stochastic process. Let $z_{v}$ be the time at which the contagion reaches $v$, and let $z_{\max }=\max _{v \in V: z_{v}<\infty} z_{v}$. If $z_{v}=\infty$ for some vertex $v \in V$, that is, the contagion does not reach $v$, we reassign $z_{v}=z_{\max }$, as described in [Wilder, 2018].

The decision maker has a budget $B$ (e.g., energy) to spend on sensing resources. Let $x_{v}$ represent the amount of energy allocated to the sensor at a vertex $v$. When contagion reaches $v$ at time $z_{v}$, the sensor detects it with probability $1-(1-p)^{x_{v}}$, where $p \in[0,1]$ is the probability that detects the contagion per unit of energy. The objective $F$ on vectors $\boldsymbol{x}=\left(x_{v}\right)_{v \in V}$ and $\boldsymbol{z}=\left(z_{v}\right)_{v \in V}$ is the expected amount of detection time that is saved by the sensor placements:

$$
F(\boldsymbol{x} ; \boldsymbol{z})=z_{\max }-\sum_{i=1}^{n} z_{v_{i}}\left(1-(1-p)^{x_{v_{i}}}\right) \prod_{j<i}(1-p)^{x_{v_{j}}},
$$

where the vertices are ordered so that $z_{v_{1}} \leq z_{v_{2}} \leq \cdots \leq z_{v_{n}}$. It is known that the function $F$ is DR-submodular [Bian et al., 2017].

Datasets. We consider two sensing models and generated three datasets. In all of them, the source vertex $s$ is chosen uniformly at random.

The first model is the continuous time independent cascade model (CTIC). In this model, each edge $u v \in E$ has propagation time $\rho_{u v}$ drawn from an exponential distribution with mean $\lambda$. The contagion starts at the source vertex $s$, i.e., $z_{s}=0$, and we iteratively set $z_{v}=\min _{u \in N(v)} z_{u}+\rho_{u v}$, where $N(v)$ is the set of neighbors of $v$. Note that $z_{v}$ is the first time that the contagion reaches $v$ from its neighbor. We generated datasets using two real-world networks ${ }^{2}$ : NetScience, a collaboration network of 1,461 network scientists, and EuroRoad, a network of 1,174 European cities and

\footnotetext{
${ }^{2}$ http://konect.cc
} 


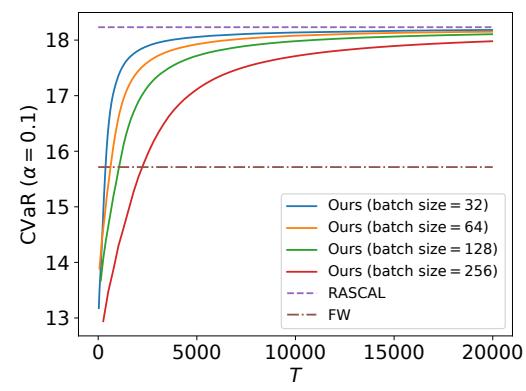

(a) NetScience

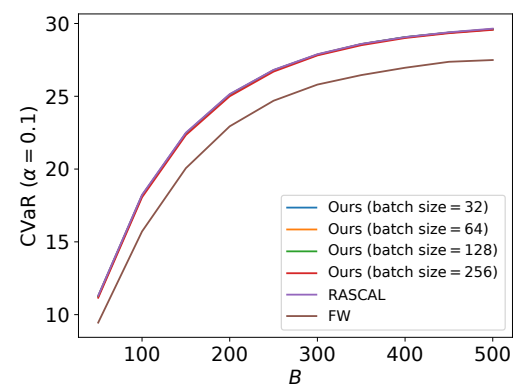

(a) NetScience

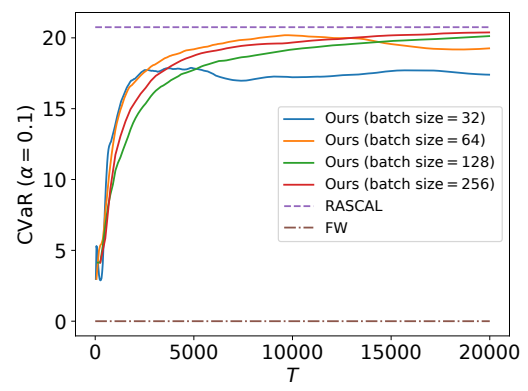

(b) EuroRoad

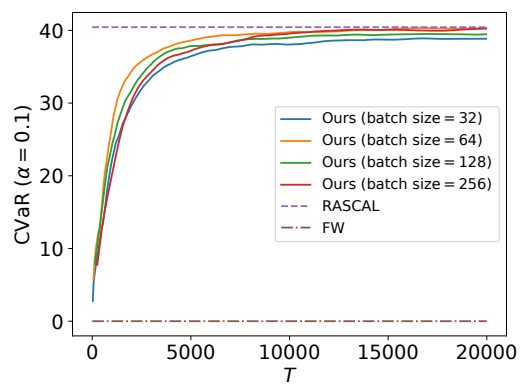

(c) BWSN

Figure 1: CVaR and the number of samples $T$

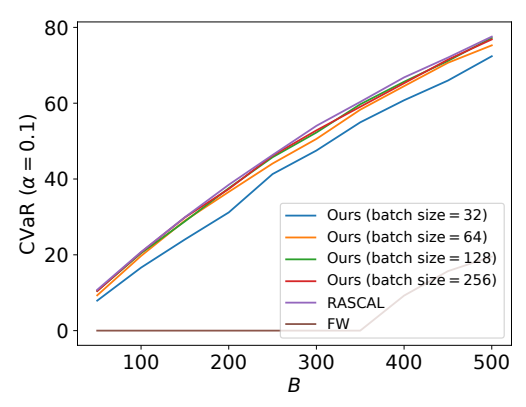

(b) EuroRoad

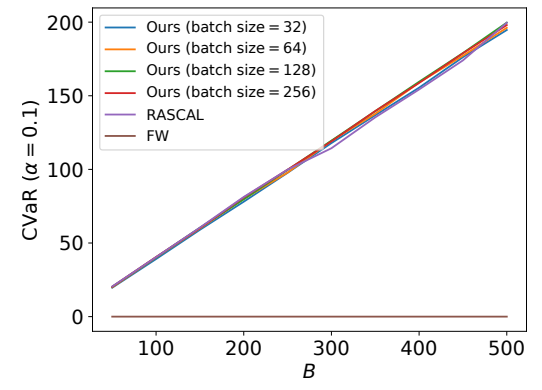

(c) BWSN

Figure 2: CVaR and budget $B$

the roads between them. For both networks, we set $\lambda=5$ and $p=0.01$, and we generated 1,000 scenarios.

The second model, known as the Battle of Water Sensor Networks (BWSN) [Ostfeld and others, 2008], involves contamination detection in a water network. BWSM simulates the spread of contamination through a 126-vertex water network consisting of junctions, tanks, pumps, and the links between them, and the $z_{v}$ values are provided by a simulator. We set $p=0.001$ and generated 1,000 scenarios.

Methods. We compared our method against two offline algorithms, RASCAL [Wilder, 2018] and the Frank-Wolfe (FW) algorithm [Bian et al., 2017]. We note that the latter algorithm is designed to maximize the expectation of a DRsubmodular function instead of its $\mathrm{CVaR}$. We run those offline methods on the generated 1,000 scenarios for each dataset. As our method is an online algorithm, we run our method on 20,000 samples in an online manner, where each sample was uniformly drawn from the set of generated scenarios.

Results. Figure 1 shows how the CVaR changes as $T$ increases. For each dataset, as long as the batch size is not excessively small, the $\mathrm{CVaR}$ attained by our method approaches to that attained by RASCAL. FW algorithm showed significantly lower performance because it is not designed to maximize CVaR.

Figure 2 shows how the CVaR changes as the budget $B$ increases. For our method, we plotted the CVaR after processing 10,000 samples. We can again confirm that the CVaR attained by our method is close to that attained by RASCAL.

\section{Acknowledgments}

T.S. is supported by JST, ERATO, Grant Number JPMJER1903, Japan. Y.Y. is supported in part by JSPS KAKENHI Grant Number 18H05291 and 20H05965.

\section{References}

[Anari et al., 2019] N. Anari, N. Haghtalab, S. Naor, S. Pokutta, M. Singh, and A. Torrico. Structured robust submodular maximization: Offline and online algorithms. In AISTATS, volume 89, 2019.

[Bian et al., 2017] A. Bian, B. Mirzasoleiman, J. Buhmann, and A. Krause. Guaranteed non-convex optimization: Submodular maximization over continuous domains. In AISTATS, pages 111-120, 2017.

[Buchbinder and Feldman, 2018] N. Buchbinder and M. Feldman. Submodular functions maximization problems. In Handbook of Approximation Algorithms and Metaheuristics, 2018.

[Calinescu et al., 2011] G. Calinescu, C. Chekuri, M. Pál, and J. Vondrák. Maximizing a monotone submodular function subject to a matroid constraint. SIAM J. Comput., 40(6):1740-1766, 2011.

[Cardoso and Xu, 2019] A. R. Cardoso and H. Xu. Riskaverse stochastic convex bandit. In AISTATS, pages 39-47, 2019. 
[Chekuri et al., 2010] C. Chekuri, J. Vondrák, and R. Zenklusen. Dependent randomized rounding via exchange properties of combinatorial structures. In FOCS, pages 575-584, 2010.

[Chen et al., 2017] R. Chen, B. Lucier, Y. Singer, and V. Syrgkanis. Robust optimization for non-convex objectives. In NIPS, pages 4705-4714, 2017.

[Chen et al., 2018] L. Chen, H. Hassani, and A. Karbasi. Online continuous submodular maximization. In AISTATS, pages 1896-1905, 2018.

[Fujishige, 2005] S. Fujishige. Submodular Functions and Optimization. Elsevier, 2nd edition, 2005.

[Golovin et al., 2014] D. Golovin, A. Krause, and M. Streeter. Online submodular maximization under a matroid constraint with application to learning assignments. arXiv, 2014.

[Hassani et al., 2017] H. Hassani, M. Soltanolkotabi, and A. Karbasi. Gradient methods for submodular maximization. In NIPS, pages 5841-5851, 2017.

[Hazan, 2016] E. Hazan. Introduction to online convex optimization. Foundations and Trends® in Optimization, 2(34):157-325, 2016

[Karbasi et al., 2019] A. Karbasi, H. Hassani, A. Mokhtari, and Z. Shen. Stochastic continuous greedy++: When upper and lower bounds match. In NeurIPS, pages 1308713097, 2019.

[Karimi et al., 2017] M. R. Karimi, M. Lucic, H. Hassani, and A. Krause. Stochastic submodular maximization: The case of coverage functions. In NIPS, 2017.

[Kempe et al., 2003] D. Kempe, J. Kleinberg, and É. Tardos. Maximizing the spread of influence through a social network. In $K D D$, pages 137-146, 2003.

[Krause and Golovin, 2014] A. Krause and D. Golovin. Submodular function maximization. In Tractability: Practical Approaches to Hard Problems, pages 71-104. Cambridge University Press, 2014.

[Krause et al., 2008] A. Krause, B. McMahan, C. Guestrin, and A. Gupta. Robust submodular observation selection. JMLR, 9:2761-2801, 2008.

[Krokhmal et al., 2002] P. Krokhmal, J. Palmquist, and S. Uryasev. Portfolio optimization with conditional valueat-risk objective and constraints. Journal of Risk, 4:43-68, 2002.

[Leskovec et al., 2007] J. Leskovec, A. Krause, C. Guestrin, C. Faloutsos, J. VanBriesen, and N Glance. Cost-effective outbreak detection in networks. In $K D D$, pages $420-429$, 2007.

[Maehara, 2015] T. Maehara. Risk averse submodular utility maximization. Operations Research Letters, 43(5):526 $529,2015$.

[Mansini et al., 2007] R. Mansini, W. Ogryczak, and G. Speranza. Conditional value at risk and related linear programming models for portfolio optimization. Annals of Operations Research, 152(1):227-256, 2007.
[Mokhtari et al., 2018] A. Mokhtari, H. Hassani, and A. Karbasi. Conditional gradient method for stochastic submodular maximization: Closing the gap. In AISTATS, pages 1886-1895, 2018.

[Ohsaka and Yoshida, 2017] N. Ohsaka and Y. Yoshida. Portfolio optimization for influence spread. In $W W W$, pages 977-985, 2017.

[Ostfeld and others, 2008] A. Ostfeld et al. The battle of the water sensor networks (bwsn): A design challenge for engineers and algorithms. J. Water Resour. Plan. Manag., 134(6):556-568, 2008.

[Rockafellar and others, 2000] T. Rockafellar et al. Optimization of conditional value-at-risk. Journal of Risk, $2: 21-42,2000$.

[Roughgarden and Wang, 2018] T. Roughgarden and J. R. Wang. An optimal algorithm for online unconstrained submodular maximization. In COLT, pages 1307-1325, 2018.

[Shapiro et al., 2014] A. Shapiro, D. Dentcheva, and A. Ruszczyński. Lectures on stochastic programming: modeling and theory. SIAM, 2014.

[Soma and Yoshida, 2015] T. Soma and Y. Yoshida. A generalization of submodular cover via the diminishing return property on the integer lattice. In NIPS, pages 847-855, 2015.

[Soma, 2019] T. Soma. No-regret algorithms for online $k$ submodular maximization. In AISTATS, pages 1205-1214, 2019.

[Staib et al., 2019] M. Staib, B. Wilder, and S. Jegelka. Distributionally robust submodular maximization. In $A I S$ TATS, volume 89, pages 506-516, 2019.

[Streeter and Golovin, 2008] M. Streeter and D. Golovin. An online algorithm for maximizing submodular functions. In NIPS, pages 1577-1584, 2008.

[Streeter et al., 2009] M. Streeter, D. Golovin, and A. Krause. Online learning of assignments. In NIPS, pages 1794-1802, 2009.

[Tamar et al., 2015] A. Tamar, Y. Glassner, and S. Mannor. Optimizing the CVaR via sampling. In $A A A I, 2015$.

[Vondrák, 2013] J. Vondrák. Symmetry and approximability of submodular maximization problems. SIAM J. Comput. 42(1):265-304, 2013.

[Wilder, 2018] B. Wilder. Risk-sensitive submodular optimization. In $A A A I$, pages 6451-6458, 2018.

[Yau et al., 2011] S. Yau, R. H. Kwon, J. S. Rogers, and D. Wu. Financial and operational decisions in the electricity sector: Contract portfolio optimization with the conditional value-at-risk criterion. Int. J. Prod. Econ, 134(1):67-77, 2011.

[Zhang et al., 2019] M. Zhang, L. Chen, H. Hassani, and A. Karbasi. Online continuous submodular maximization: From full-information to bandit feedback. In NeurIPS, volume 32, pages 9210-9221, 2019. 